Service social

\title{
Les stratégies de défense collective des droits au Québec : discours et pratiques
}

\section{Méric Sauvé et Ysabel Provencher}

Volume 64, numéro 1, 2018

URI : https://id.erudit.org/iderudit/1055889ar

DOI : https://doi.org/10.7202/1055889ar

Aller au sommaire du numéro

Éditeur(s)

École de service social de l’Université Laval

ISSN

1708-1734 (numérique)

Découvrir la revue

Citer cet article

Sauvé, M. \& Provencher, Y. (2018). Les stratégies de défense collective des droits au Québec : discours et pratiques. Service social, 64(1), 30-46.

https://doi.org/10.7202/1055889ar
Résumé de l'article

Cet article vise à mettre en lumière les résultats d'une étude sur les stratégies des organismes communautaires de défense collective de droits au Québec. D'abord, le cadre d'analyse en trois stratégies, soit émancipatoire, coopération-persuasion et confrontation sera présenté. L’importance des pratiques et les discours des organismes sur chacune des stratégies seront présentés pour avoir une vision actuelle des activités des organismes communautaires en défense collective de droits. Enfin, des liens seront faits entre les caractéristiques des organismes et leur choix de stratégie afin de déterminer si certaines caractéristiques influencent le recours à une stratégie plutôt qu'à une autre. 


\title{
Les stratégies de défense collective des droits au Québec : discours et pratiques
}

\author{
SAUVÉ, Méric, B. Ph. et M. Serv. soc. \\ Coordonnateur d'un organisme en défense collective des droits \\ PROVENCHER, Ysabel, Ph. D., D. Serv. soc., professeure titulaire \\ École de travail social et de criminologie \\ Université Laval
}

\section{RÉSUMÉ}

Cet article vise à mettre en lumière les résultats d'une étude sur les stratégies des organismes communautaires de défense collective de droits au Québec. D'abord, le cadre d'analyse en trois stratégies, soit émancipatoire, coopération-persuasion et confrontation sera présenté. L'importance des pratiques et les discours des organismes sur chacune des stratégies seront présentés pour avoir une vision actuelle des activités des organismes communautaires en défense collective de droits. Enfin, des liens seront faits entre les caractéristiques des organismes et leur choix de stratégie afin de déterminer si certaines caractéristiques influencent le recours à une stratégie plutôt qu'à une autre.

Mots-clés : Action sociale, Défense de droits, Stratégies, Organismes à but non lucratif (OBNL), Modèles d'intervention, Intervention communautaire, Organisation communautaire.

\begin{abstract}
This article aims to highlight the results of a study on the strategies of non-profitable organizations in the field of social advocacy in Quebec. First, the analytical framework into three strategies, emancipatory, cooperation-persuasion and confrontation, will be presented. The importance of the practices and the discourses of the organizations on each of the strategies will be presented in order to have an actual vision of the activities of the community organizations in social advocacy. Thereafter, links will be made between the characteristics of the organizations and their choice of strategy in order to determine whether some characteristics influence the use of one strategy rather than another.
\end{abstract}

Keywords: Social advocacy, Strategies, Non-profitable organization, Social work models, Community practice, Community organization. 


\section{INTRODUCTION}

Depuis plusieurs décennies, les pratiques de défense collective des droits mises en œuvre par des organisations de la société civile habitent le paysage politique et social du Québec et du Canada (Favreau, 1989 ; Blondin, Comeau et Provencher, 2012). Même si on s'entend pour dire que ces organismes ont connu leur apogée, en termes d'influence politique, durant les années 1970, et ce, dans différents domaines (logement, santé mentale, justice environnementale, chômage, assistance publique, incapacité et handicap, etc.) (Dickinson et Young, 1992), l'apport historique de ces organisations au développement social est aujourd'hui largement reconnu par les autorités publiques (Ministère de l'Emploi et de la Solidarité sociale, 2001 ; Rektor, 2002). Au Québec, la politique provinciale de reconnaissance de l'action communautaire autonome associe la défense collective des droits à l'action menée par les organismes qui effectuent « l'analyse des politiques gouvernementales et des projets de loi » et qui réalisent des activités visant à sensibiliser les autorités publiques « aux situations que vivent certains groupes de citoyennes et de citoyens » (Ministère de l'emploi et de la Solidarité sociale, 2001, p. 29). Or, malgré l'adoption de cette politique il y a plus d'une décennie, les connaissances au sujet des activités de défense des droits réalisées au jour le jour par les organismes communautaires demeurent superficielles. L'étude dont nous présentons ici une partie des résultats veut contribuer à l'avancement des connaissances sur cette question par la description des pratiques de défense collective des droits mises en œuvre par des organisations.

Nous décrivons notamment ici les stratégies les plus représentatives de l'action de ces organismes et les liens entre les stratégies déployées, leur niveau de revenu et leur domaine d'action. Nous évoquons enfin quelques pistes pour des recherches futures.

\section{Les stratégies mises en œuvre en matière de défense collective des droits : l'état des connaissances au Québec}

En 2013-2014, 312 organismes communautaires parmi les 5037 ayant reçu un soutien financier du gouvernement du Québec ont été reconnus dans la catégorie des organismes communautaires dits de défense collective des droits (OCDCD). Cette reconnaissance, fondée sur les dispositifs de la politique, s'appuie sur la réalisation d'activités d'éducation populaire, de mobilisation sociale, de représentation et/ou d'action politique non partisane par ces organismes communautaires. Si ces derniers ne représentent qu'une partie de l'ensemble de ceux recevant un soutien financier gouvernemental $(6,3 \%$ en 2013-2014), ils exercent leurs activités dans de nombreux domaines comme le logement, la santé mentale, la pauvreté, le féminisme, le handicap, les droits des personnes LGBT, etc. Pour réaliser leur mission de défense collective des droits, ces organismes utilisent des moyens variés (Reisch, 2005 ; Comeau et coll., 2008). Selon le Nouveau dictionnaire critique d'action sociale, la stratégie 1) se déploie en fonction d'une visée d'action sur le monde, 2) se réalise grâce à un certain nombre de moyens, 3) lesquels sont ordonnés de manière cohérente afin d'atteindre un but (Barreyre et Bouquet, 2006). Les connaissances actuelles montrent que l'on peut considérer ces moyens d'action ou tactiques en fonction de trois grandes stratégies de défense collective des droits: émancipatoire, de coopération-persuasion et de confrontation (Sauvé et Provencher, 2017). 
La stratégie émancipatoire est caractérisée par l'importance accordée aux activités d'éducation populaire centrées sur l'engagement social des individus (Checkoway, 2012 ; Lepage et coll., 2012 ; MEPACQ, 2000), par la recherche de rapports égalitaires entre les membres et par la promotion de la participation de tous à la vie interne des organisations (Comeau, 2012 ; Cook et Comeau, 2006 ; Sioui-Durand, 2011 ; Hardina, 2006). Plusieurs organisations s'inscrivant dans ce type de stratégie ont ainsi développé leur propre programme d'éducation populaire (Lavoie, 2012). Aux activités éducatives plus traditionnelles (conférences publiques, porte-à-porte, rassemblements, soirées d'information, groupes de discussion, etc.) s'ajoutent dorénavant d'autres activités d'information et de mobilisation par le biais des médias sociaux (Sioui-Durand, 2011). La stratégie de coopération-persuasion, pour sa part, prend appui sur le principe de la collaboration avec les autorités publiques dans le but de les convaincre du bien-fondé des demandes et des revendications portées par ces organisations dans différents domaines de la vie collective (Panet-Raymond, 1991 ; Bourque, 2008 ; Douay et Prevot, 2014). De nombreuses activités peuvent être données à voir dans ce type de stratégie, comme la promotion de la participation aux élections (Hardina, 2006), la soumission de mémoires et la sollicitation de rencontres avec des représentants des autorités gouvernementales (Greissler et Labbé, 2012 ; Hamzaoui, 2012; René, 2009). Par ailleurs, des tribunes mises en place par les autorités publiques elles-mêmes afin de promouvoir la participation citoyenne servent régulièrement de cadre pour la mise en œuvre de cette stratégie : consultations publiques, référendums, tables de concertation (Lamoureux, 2008 ; Baillergeau, 2008 ; Bessaïh, 2013). Ici, les activités se font dans une logique de collaboration en évitant la confrontation avec les autorités (Douay et Prevot, 2014). Notons que pour Bessaïh (2013), la création de «projets » d'action communautaire est caractéristique de cette stratégie. Enfin, depuis ses origines, l'action collective reposant sur la confrontation demeure incontournable dans les mouvements et les groupes de pression sur les pouvoirs (Comeau, 2007 ; Blondin, Comeau et Provencher, 2012). La stratégie de confrontation s'appuie sur le postulat que les résultats de l'action collective dépendent du rapport de force qu'une organisation est en mesure d'établir face aux détenteurs du pouvoir au sujet d'un enjeu qui la concerne. Les moyens associés à cette stratégie sont variés. Si la manifestation publique représente une tactique usuelle, les activités de confrontation peuvent revêtir des formes plus directes (actions de perturbation ou de confrontation), dans le but de procurer aux revendications et aux discours d'un groupe le maximum de visibilité (Greissler et Labbé, 2012 ; Comeau, 2012 ; Goldenberg et Proulx, 2011).

L'examen des travaux existants montre que de manière générale, les organismes ont recours à plus d'une stratégie d'action collective et que ces dernières ne sont pas indépendantes les unes des autres. Ainsi, une activité d'éducation populaire peut précéder une action directe de pression (Bessaïh, 2013) ; un groupe peut aussi conduire une manifestation publique afin de pouvoir négocier par la suite avec les autorités publiques (Greissler et Labbé, 2012). La littérature montre également que différents éléments de contexte interne influencent le recours aux stratégies, comme les limites financières, humaines et organisationnelles de l'organisation, les niveaux de conscience sociopolitique de ses membres, le type d'action que ces derniers sont prêts à entreprendre, etc. (Bessaïh, 2013 ; MEPACQ, 2011 ; René, 2009). Des éléments de contexte externe influencent également les pratiques comme la transformation des modes de participation citoyenne des jeunes adultes, la transformation des espaces publics par les médias sociaux, la transformation du rôle de l'État et son effet sur les pratiques de financement et de reddition de comptes des organismes (Checkoway, 2012 ; Martin, 2015 ; Baillergeau, 2008 ; Bourque et coll., 2007 ; Bessaïh, 2013 ; Martin et Posca, 2012 ; René, 2009 ; Goldenberg et Proulx, 2011 ; MEPAQ, 2011, 2013). 
Si plusieurs écrits font état de la diversité des pratiques de défense collective des droits menées par les organismes communautaires au Québec, il existe encore peu de recherches empiriques sur le sujet. Est-ce que les organismes de défense collective des droits privilégient une stratégie plutôt qu'une autre ? Si oui, en fonction de quels éléments de contexte interne et externe ? L'étude dont nous présentons ici les principaux résultats avait pour objectif de contribuer au développement des connaissances sur le sujet en décrivant les pratiques mises en œuvre sur le terrain par les groupes au regard des différentes stratégies de défense collective des droits.

\section{Cadre d'analyse}

Le concept de stratégie, à son niveau le plus opérationnel, renvoie aux tactiques qui représentent les moyens ou les activités singulières réalisées sur le terrain au jour le jour (Brueggemann, 2006 ; Sharp, 1973). Ainsi que l'illustre le tableau ci-dessous, les concepts de stratégie émancipatoire, de coopération-persuasion et de confrontation ont été associés à trois groupes d'indicateurs, chacun étant composé de tactiques (ou activités/moyens d'action) particulières (Sauvé et Provencher, 2017). 
Tableau 1. Stratégie d'action collective : opérationnalisation du concept

\begin{tabular}{|c|c|c|}
\hline Type de stratégie & Sous-dimensions & Indicateurs \\
\hline $\begin{array}{l}\text { Stratégie } \\
\text { émancipatoire }\end{array}$ & $\begin{array}{l}\text { Éducation } \\
\text { Pédagogie } \\
\text { Gestion participative } \\
\text { Conscientisation } \\
\text { Sensibilisation }\end{array}$ & $\begin{array}{l}\text { - Recrutement de bénévoles } \\
\text { - Formations auprès des membres/des bénévoles } \\
\text { - Sensibilisation/formation auprès de la population } \\
\text { - Communication avec les médias traditionnels } \\
\text { - Production de matériel de sensibilisation } \\
\text { - Éducation populaire } \\
\text { - Prise de parole publique } \\
\text { - Diffusion de vidéos, articles ou documents sur Internet } \\
\text { - Implication des membres dans la gestion de } \\
\text { l'organisme } \\
\text { - Évaluation participative des activités }\end{array}$ \\
\hline $\begin{array}{l}\text { Stratégie de } \\
\text { cooperation-persuasion }\end{array}$ & $\begin{array}{l}\text { Collaboration } \\
\text { Persuasion } \\
\text { Partenariat } \\
\text { Concertation } \\
\text { Coopération }\end{array}$ & $\begin{array}{l}\text { - Écriture de lettres ou mémoires aux autorités } \\
\text { - Participation à des tables de concertation ou } \\
\text { partenariat } \\
\text { - Développement d'actions en partenariat avec les } \\
\text { autorités } \\
\text { - Rencontre avec des éluEs } \\
\text { - Rencontre avec des acteurs privés } \\
\text { - Rencontre avec des institutions gouvernementales } \\
\text { - Encouragement du vote chez les membres } \\
\text { - Participation à des sommets citoyens } \\
\text { - Participation à des délibérations ou consultations } \\
\text { publiques } \\
\text { - Participation à des recherches-actions participatives }\end{array}$ \\
\hline Stratégie de confrontation & $\begin{array}{l}\text { Conflit } \\
\text { Adversaire } \\
\text { Lutte } \\
\text { Perturbation } \\
\text { Affrontement }\end{array}$ & $\begin{array}{l}\text { - Lancement de pétition } \\
\text { - Accompagnement juridique des membres } \\
\text { - Instauration d'une poursuite juridique } \\
\text { - Mettre fin à une coopération en signe de protestation } \\
\text { - Cyberpiratage : envoi massif de courriels, surcharge } \\
\text { d'un site } \\
\text { - Participation à une manifestation } \\
\text { - Organisation d'une manifestation } \\
\text { - Mise en place d'une grève } \\
\text { - Désobéissance civile visant la visibilité } \\
\text { - Désobéissance civile visant la perturbation }\end{array}$ \\
\hline
\end{tabular}

Une fois le concept de stratégie d'action collective opérationnalisé, le second défi de notre recherche consistait à garantir le recueil de données fiables à son sujet. Pour ce faire, nous avons constitué des listes d'activités et de moyens reflétant chacune des dimensions et sous-dimensions du concept de stratégie d'action collective à partir d'une compilation des travaux de Sharp (1973), Schmid, Bar et Nirel (2013) et Hardina (2006) sur les tactiques d'action collective (Sauvé et Provencher, 2017). En effet, leurs travaux montrent bien que les activités et moyens d'action collective peuvent être regroupés en trois grands groupes de tactiques, soit 1) les actions menées auprès des personnes 
marginalisées/en situation d'oppression et avec elles, 2) les activités de coopération et de persuasion et 3) les activités centrées sur l'augmentation du rapport de force'1.

D’abord, les tactiques auprès des personnes défavorisées ou marginalisées désignent toutes les tactiques dont l'objectif est d'agir sur ou avec les personnes impliquées. Cela inclut donc les formations pour ces personnes autant que leur implication dans la mise en place de toutes les étapes de la réalisation des transformations sociales. Les tactiques de coopération et de persuasion renvoient pour leur part aux activités visant à informer les autorités et à les convaincre de la nécessité d'appuyer une cause ou de prendre position face à un enjeu. Cela inclut la négociation, le recours aux tribunaux et l'utilisation des connaissances scientifiques pour appuyer les revendications d'un groupe. Finalement, les tactiques liées à la création d'un rapport de force réfèrent à toutes les activités et moyens d'action dont la logique cherche à contraindre l'opposant à une cause à se plier aux revendications d'un groupe. Ces moyens incluent les blocages, les manifestations et les actions de désobéissance civile, ceux-ci pouvant être combinés pour augmenter l'efficacité de la stratégie.

${ }^{1}$ Considérant que certaines activités peuvent s'inscrire dans toutes les stratégies dépendamment de la manière dont elles sont réalisées ou du moment où elles le sont (comme le recours aux médias traditionnels, qui peut tout aussi bien servir à informer la population d'un problème qu'à interpeller les autorités à son sujet), nous avons retenu uniquement les tactiques fortement associées à une seule stratégie de manière à réduire au maximum les ambiguïtés. 
Tableau 2 : Activités et moyens d'action selon les types de stratégies

\begin{tabular}{|c|c|c|}
\hline Stratégie émancipatoire & $\begin{array}{l}\text { Stratégie de coopération- } \\
\text { persuasion }\end{array}$ & Stratégie de confrontation \\
\hline 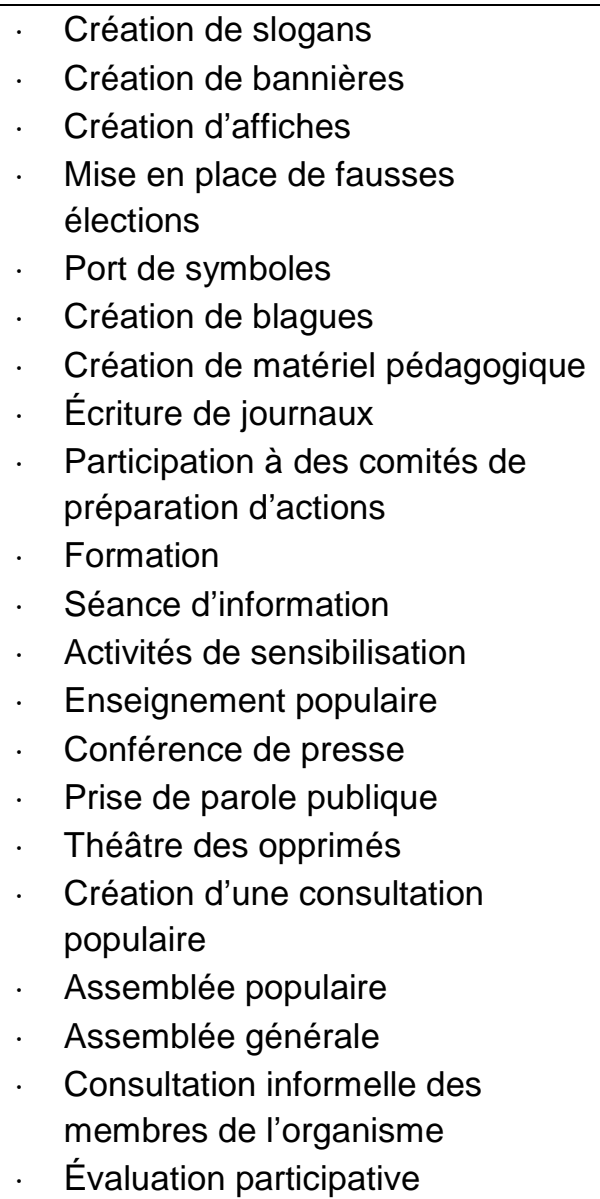 & $\begin{array}{l}\text { Rencontre avec des élus } \\
\text { Rencontre avec des acteurs } \\
\text { privés } \\
\text { Rencontre avec des } \\
\text { institutions gouvernementales } \\
\text { «Lobbying » } \\
\text { Écriture de lettre } \\
\text { Écriture de mémoire } \\
\text { Participation à des tables de } \\
\text { concertation } \\
\text { Participation à des } \\
\text { consultations publiques } \\
\text { Participation à des recherches } \\
\text { Création d'une recherche- } \\
\text { action participative } \\
\text { Vigile }\end{array}$ & 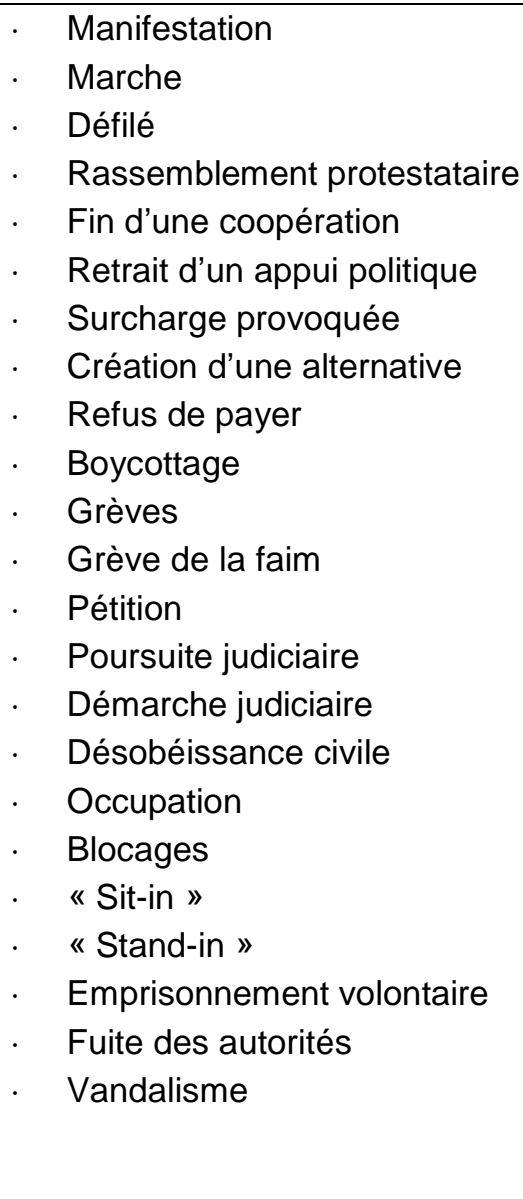 \\
\hline
\end{tabular}

Enfin, trois types de mesure ont été effectués pour illustrer le recours aux stratégies et distinguer celles caractéristiques de l'activité de différents organismes:1) les tactiques utilisées au moins une fois au cours de la dernière année, 2) les tactiques considérées par les organismes comme étant les plus représentatives de leur pratique et 3) l'importance attribuée par les répondants des organismes à chacune des stratégies. Nous avons ensuite tenté d'identifier des liens entre certaines caractéristiques de l'organisme (longévité de l'organisation, revenu annuel, domaine d'action, etc.) et le recours aux différentes stratégies.

\section{Cadre méthodologique}

Cette recherche s'inscrit dans le paradigme positiviste en continuité avec la philosophie d'Auguste Comte (Pickering, 2011). Ainsi, la recherche se voulait essentiellement descriptive et les répondants n'étaient pas considérés comme des observateurs neutres. La population à l'étude était constituée des 321 organismes ayant reçu une subvention du SACAIS en 2013-2014 à titre d'organismes 
exerçant de la défense collective des droits (SACAIS, 2015). Un échantillonnage de hasard simple (Mayer, Ouellet, Saint-Jacques et Turcotte, 2000) a été effectué à partir des listes des organismes récipiendaires du soutien financier gouvernemental dans chacune des régions administratives du Québec pour former un échantillon de 42 unités qui, sans permettre la généralisation des résultats ${ }^{2}$, pouvait servir efficacement les objectifs de l'étude. Les données ont été récoltées à l'aide de deux sources d'information : le rapport d'activités annuel des organismes participants et un questionnaire auto-administré, comportant des questions fermées, semi-ouvertes et ouvertes. Le questionnaire a permis d'obtenir des informations sur le recours à chacune des stratégies et sur différentes caractéristiques des organismes.

\section{RÉSUltats}

\section{Caractéristiques de l'échantillon}

Parmi les 170 sollicités, 42 organismes, provenant de 14 des 17 régions administratives du Québec ${ }^{3}$, ont participé à cette recherche. Un peu plus du quart des organismes interrogés $(26,2 \%)$ proviennent de la région de Montréal, ce qui constitue une proportion légèrement plus faible que dans la population à l'étude (32,4\%). Les organismes participants existent en moyenne depuis 32,6 ans. Ainsi qu'on peut le voir au tableau suivant, la pauvreté ${ }^{4}$ et les situations de handicap ${ }^{5}$ figurent parmi les domaines d'action principaux, totalisant 27 organismes, soit 64,3\% de l'échantillon.

Tableau 3 : Répartition de l'échantillon selon le domaine d'action

\begin{tabular}{|c|c|c|}
\hline Domaine d'action & $\mathbf{N}$ & $\begin{array}{c}\text { Répartition de } \\
\text { l'échantillon (\%) }\end{array}$ \\
\hline Pauvreté & 13 & 31 \\
\hline Handicap & 14 & 33,3 \\
\hline Logement & 4 & 9,5 \\
\hline Gérontologie & 3 & 7,1 \\
\hline Féminisme & 2 & 4,8 \\
\hline LGBTQ & 2 & 4,8 \\
\hline Autre $^{6}$ & 4 & 9,5 \\
\hline Total & 42 & 100 \\
\hline
\end{tabular}

\footnotetext{
2 Garantir le caractère probabiliste de l'échantillon avec une marge d'erreur de $5 \%$ et un niveau de confiance de $95 \%$ aurait demandé une collecte des données auprès de 175 unités (Mayer et coll., 2000). Considérant que cette étude a été réalisée dans le cadre d'un mémoire de maîtrise associé à des limites de temps et de budget, obtenir un tel échantillon semblait irréaliste.

3 Seules les régions de Laval, de la Côte-Nord et du Nord-du-Québec n'ont pas été couvertes par la recherche.

4 Le domaine Pauvreté regroupe les organismes qui défendent les droits des personnes en situation d'itinérance, sans emploi ou à faible revenu.

${ }^{5}$ Le domaine Handicap regroupe les organismes qui œuvrent auprès des personnes ayant des incapacités d'ordre physique ou mental.

6 Les organismes dans la catégorie « Autre » étaient les seuls dans leur domaine.
} 
La grande majorité des organismes participants $(80,5 \%)$ comptent entre un et trois employés $(80,5 \%)$ et près du tiers ont rapporté disposer d'un budget de moins de $60000 \$$ par année $(31 \%)$, tandis que sept organismes (16,6\%) disposent d'un budget de plus de 200000 \$an. Au total, un peu plus de la moitié, soit $54,8 \%$ des organismes, disposent d'un budget annuel de moins de $100000 \$$ par année. En moyenne, $72,6 \%$ du budget disponible provient du gouvernement provincial (la médiane du taux de financement provincial s'élève à $85 \%$, 15,8 \% du financement provient du secteur privé, 7,8\% provient des activités d'autofinancement et 3,8\% provient du gouvernement fédéral. Ainsi, le gouvernement provincial est de loin la principale source de revenus de ces organismes.

\section{Caractéristiques du recours aux stratégies}

\section{Stratégies le plus fréquemment mises en œuvre}

Les organismes interrogés dans le cadre de cette étude ont déclaré avoir recours à diverses tactiques (ou moyens d'action) liées à chacune des trois stratégies. Au cours de la dernière année, ils ont eu recours en moyenne à un peu plus de huit tactiques associées à la stratégie émancipatoire, à un peu plus de six tactiques associées à la coopération-persuasion et à près de trois tactiques de confrontation. En somme, les organismes rapportent l'utilisation d'une plus grande variété de tactiques dans la stratégie émancipatoire que dans la stratégie de coopération-persuasion. Les tactiques associées à la stratégie de coopération-persuasion mises en œuvre au cours de la dernière année étaient aussi plus diversifiées que celles de la stratégie de confrontation. Selon les répondants, les tactiques le plus fréquemment utilisées sont, dans l'ordre, la formation et la sensibilisation auprès de la population, la formation auprès des membres et des bénévoles, la production de matériel de sensibilisation et la participation à des tables de concertation. De ces quatre tactiques, trois sont associées à la stratégie émancipatoire, seule la participation à des tables de concertation est associée à la stratégie de coopération-persuasion.

\section{Stratégies considérées comme les plus représentatives}

Parmi les tactiques rapportées comme représentant le mieux leur pratique, 29 organismes ont fait référence à une tactique de la stratégie émancipatoire, 10 ont mentionné une tactique de la stratégie de coopération-persuasion et 2 organismes, une tactique associée à la stratégie de confrontation. Ainsi, les organismes considèrent généralement que la stratégie émancipatoire représente le mieux leur pratique. Par ailleurs, malgré la moins grande diversité rapportée dans le recours aux tactiques de confrontation, plusieurs organismes ont identifié des tactiques de confrontation parmi les tactiques les plus représentatives de leur action collective en matière de défense des droits. Six organismes ont identifié une tactique de confrontation comme étant la deuxième tactique la plus représentative de l'action menée par l'organisation et sept autres l'ont inscrite au troisième rang des tactiques les plus représentatives de leur pratique.

\section{Stratégies considérées comme les plus pertinentes}

Nous avons sollicité l'opinion des répondants des organismes au sujet de la nécessité ou de la pertinence des stratégies en leur demandant d'associer un score sur une échelle de 0 à 10 (0 
signifiant « aucunement nécessaire » et 10 signifiant “absolument nécessaire ») à chacune des trois stratégies ${ }^{7}$. Solliciter ces opinions nous semblait pertinent pour valider les réponses concernant les stratégies les plus fréquentes et les plus représentatives. Des écarts entre les résultats à ces questions auraient pu laisser croire que les stratégies employées étaient fortement influencées par des facteurs externes aux organismes. Au total, la stratégie émancipatoire obtient un score de 9,24/10, la stratégie de coopération-persuasion, de 7,48/10 et la stratégie de confrontation, de 5,6/10. Ici encore, on constate que les trois stratégies sont majoritairement considérées nécessaires en matière de défense collective des droits. En outre, l'ordonnancement entre les stratégies demeure la même que pour les indicateurs précédents ; la stratégie émancipatoire est considérée comme la plus pertinente ou nécessaire, suivie de la stratégie de coopération-persuasion et de la stratégie de confrontation.

La pertinence de la stratégie émancipatoire semble faire l'unanimité au sein des organismes interrogés. Un seul d'entre eux a inscrit une note inférieure à 7 et 26 organismes sur 42 ont inscrit la note maximale. Ainsi, presque tous les organismes affirment que la stratégie émancipatoire est extrêmement pertinente et importante à leurs yeux, comme l'illustrent certaines réponses au questionnaire. "Les activités de sensibilisation sont essentielles pour remplir notre mission » et "L'un des volets essentiels de notre action est l'éducation populaire". D'autres répondants mentionnent que, sans la stratégie émancipatoire, il leur serait difficile de réaliser leur mission puisqu'il ne serait pas possible de connaître les besoins des personnes visées par l'organisme. Un répondant affirme à cet égard que "sans la participation des membres, [notre organisme] perd son sens ", tandis qu'un autre affirme que "c'est à partir du vécu [des personnes] [...] que nous pouvons mieux connaître leurs réalités et leurs besoins". Plusieurs organismes mentionnent également l'identité de leur organisme pour expliquer l'importance des stratégies émancipatoires dans leur pratique: "Ces éléments sont [...] l'essence même de ce que nous sommes" et "C'est l'identité de notre organisme ». Par conséquent, plusieurs organismes semblent associer assez directement la stratégie émancipatoire avec ce qu'est la défense collective de droits en tant que telle.

\footnotetext{
7 Afin d'orienter les participants sur la définition qu'on accordait à chacune des stratégies, les questions contenaient les sous-dimensions de chacune des stratégies. (Ex: Sur une échelle de 1 (aucunement nécessaire) à 10 (absolument nécessaire), dans quelle mesure votre organisme considère-t-il que les activités d'éducation, de formation, de sensibilisation et de participation des membres à la gestion de l'organisme soient nécessaires à l'atteinte des objectifs de l'organisme ?)
} 
Quant au discours lié à la pertinence de la stratégie de coopération-persuasion, les résultats rendent compte d'opinions plus diversifiées. En effet, les organismes sont beaucoup moins nombreux que dans le cas de la stratégie émancipatoire à avoir accordé une note de 10 à cette stratégie et 11 organismes lui ont attribué une note de 6 ou moins. Plusieurs répondants ont mentionné qu' "une collaboration et une bonne communication entre l'organisme et les autorités est essentielle » et qu' "il est impératif que nous collaborions avec les instances décisionnelles ". Néanmoins, d'autres semblent associer cette stratégie à une contrainte de nécessité : "Ce sont eux qui font les règles, alors nous devons les influencer » et estiment qu'il est "difficile de faire autrement ». Par ailleurs, plusieurs répondants ont indiqué qu'ils considèrent cette stratégie préférable ou souhaitable, mais qu'elle n'est pas toujours réaliste ou efficace: "dans un monde idéal, [la collaboration est] absolument nécessaire, mais nous arrivons à travailler à l'atteinte de nos objectifs même si ce n'est pas le cas": "dans la réalité, nous avons peu ou pas d'écoute et l'impression de perdre notre temps ».

Enfin, les résultats obtenus sur l'importance accordée à la stratégie de confrontation sont encore plus variés. Quatorze organismes sur 42 ont accordé un score inférieur à 5/10 quant au degré de nécessité de cette stratégie, 19 autres lui ont accordé un score supérieur à 5, alors que 9 ont accordé le score de 5. Les résultats obtenus font voir des visions relativement divergentes sur la nécessité ou la pertinence accordée à cette stratégie d'action collective. Pour certains répondants, cette stratégie est considérée comme non nécessaire, voire comme nuisible pour l'organisme. Un répondant affirme à ce sujet que la confrontation ne «donne aucun résultat » et un autre déclare qu'on peut très bien se faire entendre tout en étant pacifique. D'autres répondants, sans tenir un discours aussi catégorique que les premiers, considèrent la stratégie de confrontation comme peu intéressante, mais estiment qu'elle reste parfois nécessaire. Ainsi, un répondant déclare: "Nous ne valorisons pas une approche de confrontation et de conflit, cependant, comme organisme de défense collective des droits, nous sommes toujours alignés sur nos valeurs » et un autre affirme : "Nous avons une bonne collaboration [...] la confrontation est rarement nécessaire, mais demeure un outil. » Selon les répondants, la confrontation n'est utile que lorsque les objectifs ne peuvent être atteints autrement. La stratégie de confrontation apparaît alors comme une stratégie de « dernier recours » comme le déclare l'un d'entre eux : "Nous n'avons bien souvent que ces solutions pour faire avancer certains dossiers. » Une autre personne indique: "Comme elles [les autorités] ne collaborent pas naturellement comme il serait idéal qu'elles le fassent, nous devons continuer à travailler dans l'intérêt général de la population [...]. C'est pourquoi le dérangement et la confrontation deviennent nécessaires. " D'autres sont plus affirmatifs sur la pertinence et la nécessité de la stratégie de confrontation pour obtenir des résultats: "Collaborer c'est bien, mais le changement n'est pas possible si l'on ne met pas notre poing sur la table " et "II faut toujours se battre [...] afin de poursuivre nos objectifs et cela demande certaines confrontations auprès des autorités pour être entendu ». Ainsi, ces derniers répondants accordent une plus grande valeur à la stratégie de confrontation, contrairement à la majorité d'entre eux. Un répondant affirme que la confrontation " va de soi pour nous, parce que c'est essentiel pour bâtir un rapport de force. La confrontation possible suffit souvent à faire bouger les choses. " Ici, la stratégie de confrontation fait partie intégrante de leur vision de la défense collective de droits et de la mission des organisations.

Ces nouvelles données permettent ainsi de mieux comprendre l'usage et la perception des différentes stratégies d'action par des organismes de défense collective des droits. Néanmoins, il est intéressant 
de se demander si ces actions et ces discours sont aussi influencés par certaines caractéristiques relatives aux organismes.

\section{Revenu disponible, domaine d'action et recours aux stratégies}

À ce sujet, les résultats indiquent que les organismes disposant de revenus plus élevés ont davantage recours à la stratégie de coopération-persuasion et n'utilisent que très peu les tactiques liées à la stratégie de confrontation. À l'inverse, les organismes qui ont un budget restreint utilisent davantage la stratégie de confrontation. Les données obtenues laissent croire à une relation entre le choix des stratégies et le revenu total des organismes étudiés. Cela semble aller dans le sens des propos de Greissler et Labbé (2012) qui soulignent que les activités de confrontation comme les actions de perturbation peuvent servir à augmenter la visibilité d'un groupe. Les groupes plus petits ayant moins de visibilité que les plus grands, il est possible qu'ils aient davantage besoin d'activités de confrontation pour se faire entendre. Néanmoins, des études supplémentaires seraient nécessaires pour démontrer et détailler cette relation.

Cela dit, la situation des organismes dont les revenus varient entre $60000 \$$ et $80000 \$$ par année semble tributaire d'une autre logique. Ces organismes identifient davantage de tactiques associées à la stratégie de coopération-persuasion que de tactiques associées aux stratégies émancipatoire et de confrontation. L'examen de la composition de cette catégorie montre que les regroupements d'associations y sont surreprésentés. On peut alors penser que le type d'organisme - selon qu'il représente une population dans un domaine d'action particulier ou qu'il est composé d'un regroupement d'organismes - exerce également une influence sur le recours aux stratégies d'action collective. À la lumière des informations sur les éléments de contexte externe pouvant influencer les activités des organismes comme la transformation du rôle de l'État et son effet sur les pratiques de financement et de reddition de comptes des organismes (Dostaler, 1999 ; Martin, 2015), on peut se demander si cette division des rôles entre les organismes de membres et les regroupements d'organismes n'est pas une réaction des organismes pour s'adapter aux changements gouvernementaux, ou encore, une manière pour les plus petits organismes d'augmenter leur visibilité sans avoir à recourir à la stratégie de confrontation. Ces hypothèses demeurent à démontrer par des recherches ultérieures.

Dans leur étude sur les activités d'action sociale dans les organismes à but non lucratif, Schmid et ses collaborateurs (2008) ont démontré que plus les organismes sont dépendants financièrement du gouvernement, moins ils pratiquent la défense collective de droits et moins ils réalisent d'activités politiques. Considérant qu'au Québec, les fonds provenant du gouvernement provincial représentent une source majeure de revenus pour les organismes interrogés, nous avons cherché à déterminer s'il existe des liens entre le recours à la stratégie de confrontation et la proportion de leurs revenus provenant de fonds gouvernementaux. Or, parmi les organismes ayant indiqué la stratégie de confrontation comme stratégie la plus représentative de leur pratique, la même proportion $(7,3 \%)$ provient d'organismes financés à hauteur de $40 \%$ que d'organismes financés à plus de $99 \%$ par le gouvernement provincial. Ainsi, cela s'oppose aux résultats obtenus par Schmid et ses collaborateurs (2008). 
Enfin, on observe des variations dans le recours aux stratégies selon le domaine d'action ${ }^{8}$. La stratégie émancipatoire et la stratégie de confrontation sont identifiées comme deux stratégies dominantes par les organismes agissant dans le domaine de la pauvreté, tandis que les organismes œuvrant dans le domaine du logement sont plus nombreux à identifier la stratégie de confrontation. Par ailleurs, plus de la moitié des organismes œuvrant à la défense des droits des personnes vivant avec une situation de handicap identifient la stratégie de coopération-persuasion comme stratégie la plus représentative de leur pratique, un niveau largement supérieur à celui rapporté par les autres organismes, où le caractère représentatif de cette stratégie ne dépasse pas le seuil des $30 \%$.

\section{Conclusion}

Notre étude avait pour objectif de mieux comprendre quelles sont les stratégies utilisées par les organismes de défense collective des droits au Québec et quels liens peuvent être esquissés entre certaines caractéristiques de ces organismes et leur recours aux différentes stratégies d'action. Les résultats obtenus permettent de constater que les organismes intègrent les trois stratégies dans leur pratique. La stratégie émancipatoire occupe la place la plus importante, suivie de la stratégie de coopération-persuasion, puis de la stratégie de confrontation. Cette dernière est la seule au sujet de laquelle nous avons observé des positions opposées quant à sa nécessité et sa pertinence, même si, de manière générale, le discours de la majorité des répondants des organismes sondés accorde une valeur à chacune des trois stratégies. Cette recherche ouvre ainsi des pistes de réflexion intéressantes en soulevant quelques questions. Grosso modo, les organismes ont une vision assez semblable de la stratégie de coopération-persuasion, alors que la stratégie de confrontation est plus polémique. En outre, une portion significative des répondants a mentionné que la stratégie de confrontation constituait un dernier recours ou une pratique à n'utiliser que dans certaines situations particulières. Ainsi, cela laisse penser que plusieurs organismes favorisent le recours à la stratégie de coopération-persuasion avant celle de la confrontation. À la lumière des résultats obtenus, peut-on avancer, à titre d'hypothèse à vérifier, que les organismes œuvrant au sein des domaines "Pauvreté » et "Logement » auraient davantage recours à la stratégie de confrontation dans la mesure où leurs revendications sont moins prises en considération par les pouvoirs publics ? En d'autres mots, les difficultés que rencontrent les organismes œuvrant dans ces domaines à obtenir gain de cause au moyen d'une stratégie de coopération-persuasion pourraient-elles faire qu'ils optent plus souvent pour la stratégie de confrontation afin de «forcer » les autorités à les écouter ? Si cette explication concorde avec le sens des réponses que les organismes ont fournies sur leur recours aux stratégies et avec les combinaisons possibles entre les différentes stratégies présentés dans la littérature (Bessaïh, 2013), elle ne nous éclaire pas sur les raisons pour lesquelles les organismes des domaines "Pauvreté » et "Logement » auraient plus de difficultés que les autres à atteindre leurs objectifs en utilisant la stratégie de coopération-persuasion.

Nous sommes d'avis que ces difficultés peuvent être expliquées, en partie, par la transformation de la conception du rôle de l'État qui s'opère depuis près de deux décennies dans les pays occidentaux. Comme on sait, le néolibéralisme promeut le marché comme outil de redistribution de la richesse (Dostaler, 1999) et le rôle de l'État devient celui d'aider l'individu à intégrer le marché du travail

\footnotetext{
${ }^{8}$ Les catégories "Pauvreté » et "Handicap » regroupent beaucoup plus d'organismes que les autres catégories. Par conséquent, ces deux catégories ont davantage de chance d'être représentatives de ces domaines d'action que les catégories où il y a moins d'organismes.
} 
comme moyen principal d'autosuffisance. Considérant que les orientations du gouvernement libéral s'inspirent directement de cette idéologie (Martin, 2015), il pourrait sembler cohérent que, dans la mesure où les personnes ayant des incapacités rencontrent des obstacles majeurs relativement à leur insertion au marché du travail, l'État considère avoir une responsabilité plus grande envers ce groupe de population. Parallèlement, les pouvoirs publics seraient moins réceptifs aux besoins de soutien en matière de logement ou de situation de pauvreté, considérant que l'insertion des individus sur le marché du travail, devrait servir à répondre à ces besoins. Finalement, si les commentaires sur l'importance accordée à chacune des stratégies étaient éclairants, il serait intéressant d'en apprendre davantage sur les tactiques et les stratégies déployées par les organismes en questionnant directement les membres au sujet des facteurs qui, de leur point de vue, les amènent à choisir une stratégie plutôt qu'une autre. II est à espérer que d'autres recherches permettront d'approfondir les résultats obtenus dans cette étude et de mieux comprendre, par exemple, l'influence des relations que les organismes de défense collective des droits entretiennent avec l'État et d'autres acteurs de la société civile sur leurs activités quotidiennes. 


\section{BIBLIOGRAPHIE}

Baillergeau, É. (2008). «Intention formative, éducation populaire et intervention sociale au Québec », Savoirs, vol. $18, n^{\circ} 3$, p. 9-35.

Barreyre, J.-Y., et B. Bouquet (2006). Nouveau dictionnaire critique d'action sociale, Paris, Bayard.

Bessaïh, N. (2013). "L'R des centres de femmes du Québec ", Nouvelles questions féministes, vol. 32, no 2, p. 130-134.

Blondin, M., Y. Comeau et Y. Provencher (2012). Innover pour mobiliser. L'actualité de l'expérience de Michel Blondin, Québec, Presses de l'Université du Québec.

Bourque, D. (2008). Concertation et partenariat. Entre levier et piège du développement des communautés, Québec, Presses de l'Université du Québec.

Bourque, D., Y. Comeau, L. Favreau et L. Fréchette (2007). L'organisation communautaire. Fondements, approches et champs de pratique, Québec, Presses de l'Université du Québec.

Brueggemann, W. G. (2006). The Practice of Macro Social Work ( $3^{e}$ éd.), Belmont, Thomson Higher Education.

Checkoway, B. (2012). «Education for democracy by young people in community-based organizations », Youth \& Society, vol. 45, n 3, p. 389-403.

Comeau, Y. (2007). « L'approche d'action sociale ou sociopolitique en organisation communautaire ", dans D. Bourque, Y. Comeau, L. Favreau et L. Fréchette, L'organisation communautaire. Fondements, approches et champs de pratique, Québec, Presses de l'Université du Québec, p. 81100.

Comeau, Y. (2012). "Les stratégies d'intervention sociopolitique favorables à l'action collective ", Reflets : Revue d'intervention sociale et communautaire, vol. 18, nº 1, p. 61-90.

Comeau, Y., M. Duperré, Y. Hurtubise, C. Mercier et D. Turcotte (2008). « Phénomènes d'influence sur la structuration de l'organisation communautaire au Québec », Service social, vol. 54, n 1 , p. 7-22.

Cook, M., et Y. Comeau (2006). "Les stratégies d'intervention sociopolitique en organisation communautaire : une illustration empirique ", Service social, vol. 52, n 1, p. 1-16.

Dickinson, J. A., et B. Young (1992). Brève histoire socio-économique du Québec, Sillery, Septentrion.

Dostaler, G. (1999). « Hayek et sa reconstruction du libéralisme », Cahiers de recherche sociologique, vol. 32, p. 119-141.

Douay, N., et M. Prevot (2014). «Park(ing) Day : label international d'un activisme édulcoré ? ", Environnement urbain, vol. 8, p. 14-33. 
Favreau, L. (1989). Mouvement populaire et intervention communautaire de 1960 à nos jours. Continuités et ruptures, Montréal, Éditions du Fleuve.

Goldenberg, A., et S. Proulx (2011). «Political action in regard to information and communication technologies », Globe : Revue internationale d'études québécoises, vol. 14, n 1, p. 99-120.

Greissler, É., et F. Labbé (2012). « Lutter pour exister », Nouvelles pratiques sociales, vol. 25, n 1, p. 208-223.

Hamzaoui, F. (2012). Le militantisme vert au Québec à l'ère d'Internet : analyse du cadrage dans les messages de Greenpeace, Trois-Rivières, Université du Québec à Trois-Rivières.

Hardina, D. (2006). « Strategies for citizen participation and empowerment in non-profit, communitybased organizations », Community Development, vol. 37, n 4, p. 4-17.

Lamoureux, D. (2008). “Démocratiser radicalement la démocratie », Nouvelles pratiques sociales, vol. $21, \mathrm{n}^{\circ} 1$, p. $121-136$.

Lavoie, C. (2012). « Race, power and social action in neighborhood community organizing: Reproducing and resisting the social construction of the other », Journal of Community Practice, vol. $20, n^{\circ} 3$, p. 241-259.

Lepage, F., et coll. (2012). Éducation populaire, une utopie d'avenir, Paris, Les liens qui libèrent.

Martin, É. (2015). Le projet « austéritaire ». La " révolution » néolibérale de l'État, Montréal, IRIS. http://iris-recherche.s3.amazonaws.com/uploads/publication/file/Fourth_Revolution_WEB.pdf

Martin, É., et J. Posca (2012). Du néolibéralisme, vraiment, Montréal, IRIS. http://irisrecherche.qc.ca/blogue/du-neoliberalisme-vraiment/

Mayer, R., F. Ouellet, M.-C. Saint-Jacques et D. Turcotte (2000). Méthodes de recherche en intervention sociale, Boucherville, Gaëtan Morin.

MEPACQ (2000). Bulletin du Mouvement d'éducation populaire et d'action communautaire du Québec, n 15. http://collections.banq.qc.ca/ark:/52327/bs1926217

MEPACQ (2011). Le Livre noir des organismes en défense collective des droits. http://www.mepacq.qc.ca/wp-content/uploads/2011/11/LIVRE_NOIRVF.pdf

MEPACQ (2013). L’ACA dénaturée ! Les groupes en DCD laissés-pour-compte ! http://trovepo.org/documents/FeuilletDCDversionlarge3.pdf

Ministère de l'Emploi et de la Solidarité sociale (2001). L'action communautaire. Une contribution essentielle à l'exercice de la citoyenneté et au développement social du Québec, Québec, MESS. https://www.mess.gouv.qc.ca/telecharger.asp?fichier=/publications/pdf/SACA_politique.pdf

Panet-Raymond, J. (1991). Partenariat ou Pater-nariat ?, Montréal, École de service social. 
Pickering, M. (2011). «Le positivisme philosophique : Auguste Comte », Revue interdisciplinaire d'études juridiques, vol. 67, n², p. 49-67.

Reisch, M. (2005). «Radical community organizing », dans M. Weil, M. Reisch et D. N. Gamble, The Hanbook of Community Practice, Thousand Oaks et Londres, SAGE Publications, p. 287-304.

Rektor, L. (2002). L'action sociale ou la défense collective des droits. La voix des citoyens, Ottawa, ISBC. http://www.vsi isbc.ca/fr/policy/pdf/position_paper.pdf

René, J.-F. (2009). «L'individualisation de l'intervention dans les organismes communautaires : levier ou barrière à la prise en charge démocratique ? », Nouvelles pratiques sociales, vol. $22, \mathrm{n}^{\circ} 1$, p. 111-124.

Secrétariat à l'action communautaire autonome et aux initiatives sociales (SACAIS) (2015). Soutien financier gouvernemental en action communautaire. État de situation consolidé 2012-2013 20132014, Québec, Ministère du Travail, de l’Emploi et de la Solidarité sociale.

Sauvé, M., et Y. Provencher (2017). « Défense collective des droits : des modèles d'intervention aux stratégies d'action », Reflets : Revue d'intervention sociale et communautaire, vol. 23, n 1, p. 5781.

Schmid, H., M. Bar et R. Nirel (2008). « Advocacy activities in nonprofit human service organizations: Implications for policy », Nonprofit and Voluntary Sector Quarterly, vol. 37, n 4, p. 581-603.

Sharp, G. (1973). The politics of Nonviolent Action, Boston, Porter Sargent.

Sioui-Durand, G. (2011). « L'art politique : nouvelles ruses et anarchie », Inter, art actuel, n 107, hiver, p. 16-29. 